\title{
Normal and skewed phosphorene nanoribbons in combined magnetic and electric fields
}

\author{
Vladimir V. Arsoski, ${ }^{1, *}$ Marko M. Grujić, ${ }^{1,2, \dagger}$ Nemanja A. Čukarić, ${ }^{1,2, \ddagger}$ Milan Ž. Tadić, ${ }^{1, \S}$ and François M. Peeters ${ }^{2, \|}$ \\ ${ }^{1}$ School of Electrical Engineering, University of Belgrade, P.O. Box 3554, 11120 Belgrade, Serbia \\ ${ }^{2}$ Department of Physics, University of Antwerp, Groenenborgerlaan 171, B-2020 Antwerp, Belgium \\ (Received 11 April 2017; revised manuscript received 29 July 2017; published 25 September 2017)
}

\begin{abstract}
The energy spectrum and eigenstates of single-layer black phosphorus nanoribbons in the presence of a perpendicular magnetic field and an in-plane transverse electric field are investigated by means of a tight-binding method, and the effect of different types of edges is examined analytically. A description based on a continuum model is proposed using an expansion of the tight-binding model in the long-wavelength limit. The wave functions corresponding to the flatband part of the spectrum are obtained analytically and are shown to agree well with the numerical results from the tight-binding method for both narrow $(10 \mathrm{~nm})$ and wide $(100 \mathrm{~nm})$ nanoribbons. Analytical expressions for the critical magnetic field at which Landau levels are formed and the ranges of wave numbers in the dispersionless flatband segments in the energy spectra are derived. We examine the evolution of the Landau levels when an in-plane lateral electric field is applied, and we determine analytically how the edge states shift with magnetic field. For wider nanoribbons, the conductance is shown to have a characteristic staircase shape in combined magnetic and electric fields. Some of the stairs in zigzag and skewed armchair nanoribbons originate from edge states that are found in the band gap.
\end{abstract}

DOI: 10.1103/PhysRevB.96.125434

\section{INTRODUCTION}

Phosphorene, a single layer of black phosphorus, is a recently isolated material [1-4] that provides some extraordinary advantages over other two-dimensional (2D) materials. Most importantly, it has a large band gap of about $1.84 \mathrm{eV} \mathrm{[5],}$ thus circumventing the main drawback of graphene with its zero band gap. Additionally, because of the puckered structure $[2,6,7]$, it has highly anisotropic electronic and thermal properties, allowing for the emergence of new functionalities. In addition, higher carrier mobilities can be achieved as compared to the $2 \mathrm{D}$ materials consisting of transition-metal dichalcogenides [8].

However, phosphorene also has two major downsides: (i) the quality of the samples degrades with time when exposed to air [9], and (ii) unlike few-layer phosphorene, the band gap is not electrically tunable. The first issue can be evaded successfully by, for instance, encapsulating phosphorene with hexagonal boron nitride, as was done previously with graphene [10-12]. Besides strain [13,14], fashioning phosphorene into nanoribbons can modify the band gap through the quantum confinement effect. Indeed, several theoretical papers have studied various types of phosphorene nanoribbons, and all of them show the potential for certain applications [15-17]. Note that due to the anisotropy of phosphorene, there are two distinct types of zigzag (ZZ) and armchair (AC) edges-normal and skewed - with skewed edges intersecting the ridges of phosphorus atoms at a sharp angle [15]. Intriguingly, normal zigzag and skewed armchair (sAC) nanoribbons are metallic, while skewed zigzag (sZZ) and normal armchair nanoribbons are insulating, and all of them have an electrically tunable band

\footnotetext{
*vladimir.arsoski@etf.bg.ac.rs

†marko.grujic@etf.bg.ac.rs

${ }^{\ddagger}$ nemanja.cukaric@etf.bg.ac.rs

$\S$ milan.tadic@etf.bg.ac.rs

"francois.peeters@uantwerpen.be
}

structure, featuring metal-insulator transitions for a sufficiently strong electric field. Furthermore, as a consequence of low scattering rates of carriers, high values of electron mobility in phosphorene were demonstrated [2,3].

While it was predicted that "bulk phosphorene" displays linearly dispersing Landau levels (LLs) for low-energy quasiparticles in low magnetic fields [18-21], which was confirmed by recent experiments $[11,12]$, the results concerning the magnetic response of phosphorene nanoribbons are relatively scarce. In Ref. [18], the formation of LLs in normal nanoribbons was reported, while in Ref. [20] the impact of a magnetic field on the quasiflat bands (QFBs) of zigzag nanoribbons was investigated. The purpose of this paper is to examine the influence of a magnetic field on phosphorene nanoribbons in more detail. In particular, we will examine the band structure of phosphorene nanoribbons with various edge types (including skewed). We will discuss the impact of dispersion anisotropy on LL formation from both a qualitative and a quantitative point of view. We will also briefly consider the effect of an in-plane electric field on the LLs. The influence of the nanoribbon width on the electronic structure and transport properties will also be analyzed.

\section{THEORETICAL MODEL}

We model the phosphorene nanoribbons using the tightbinding model, which is defined by 10 hopping parameters [5] shown in Fig. 1. Within the tight-binding approximation, the Hamiltonian reads

$$
H=\sum_{i \neq j} t_{i j} e^{i \varphi_{i j}} c_{i}^{\dagger} c_{j},
$$

where the summation runs over all the lattice sites of phosphorene, $t_{i j}$ are the hopping parameters, $\varphi_{i j}=\frac{e}{\hbar} \int_{\mathbf{r}_{\mathbf{j}}}^{\mathbf{r}_{\mathbf{i}}} \mathbf{A} \cdot d \mathbf{l}$ denotes the Peierls phase picked up while hopping in the presence of the magnetic field, and $c_{j}^{\dagger}\left(c_{i}\right)$ is the creation (annihilation) operator of an electron on the site $j(i)$. 
(a)

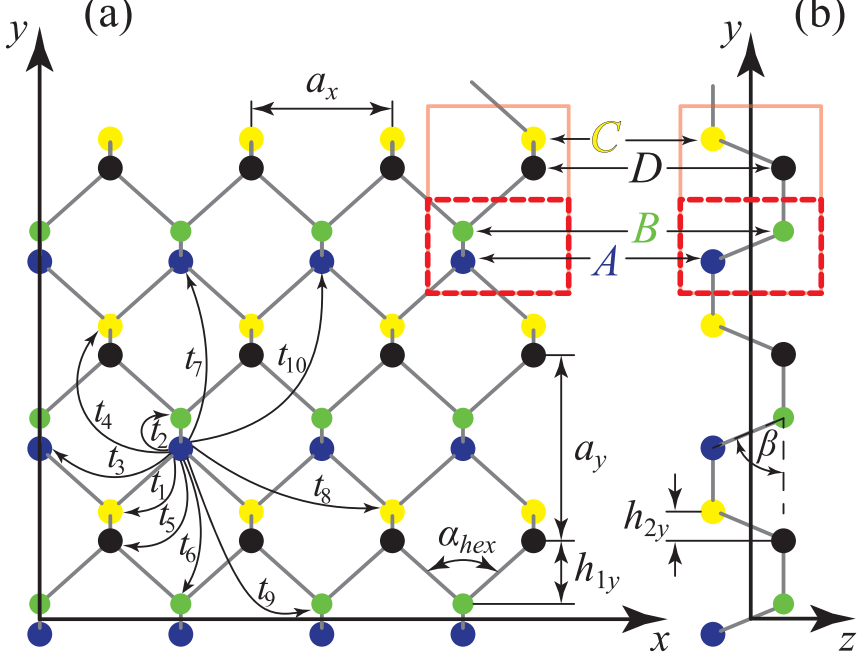

FIG. 1. Illustration of the phosphorene structure: (a) top view and (b) side view. The red transparent-solid (opaque-dashed) rectangle indicates the unit cell in the four-band (two-band) model. Relevant parameters are explicitly indicated in the figure.

The unit cell of the single phosphorene sheet, framed by the solid square line in Fig. 1, contains four atoms labeled by $A$ (blue), $B$ (green), $C$ (yellow), and $D$ (black). It is straightforward to show that the diagonal terms of the effective Hamiltonian, related to the conduction and valence band, are

$$
\begin{aligned}
& E_{c}^{\mathrm{eff}}=E_{c 0}+\frac{\hbar^{2}}{2 m_{e, x}^{*}} k_{x}^{2}+\frac{\hbar^{2}}{2 m_{e, y}^{*}} k_{y}^{2}, \\
& E_{v}^{\mathrm{eff}}=E_{v 0}-\frac{\hbar^{2}}{2 m_{h, x}^{*}} k_{x}^{2}-\frac{\hbar^{2}}{2 m_{h, y}^{*}} k_{y}^{2},
\end{aligned}
$$

respectively. Details of the derivation are given in the Appendix. Here, the effective masses along the $\Gamma-X$ direction are the same as in the case when coupling between the conduction and the valence band is neglected,

$$
m_{e(h), x}^{*}=m_{0 e(0 h), x}^{*}=\frac{\hbar^{2}}{2\left(\alpha_{x} \pm \chi_{x}\right)},
$$

while the effective masses along the $\Gamma-Y$ direction are modified by the term that perturbatively takes into account the interband coupling,

$$
m_{e(h), y}^{*}=\frac{\hbar^{2}}{2\left(\alpha_{y} \pm \chi_{y}+\frac{\gamma^{2}}{E_{g}}\right)} .
$$

The upper "+" (lower "-") sign is for the electron (hole). The effective masses along the main axes (see Fig. 1) are given in Table I. Note that there is a difference between our results and the one obtained from the previously derived continuum model [18] based on five hopping parameters [22].

Our goal is to analyze nanoribbons with arbitrary edges, where the translation vector of a unit cell is $\mathbf{d}=c_{x} a_{x} \mathbf{e}_{x}+$ $c_{y} a_{y} \mathbf{e}_{y}$. Here, $c_{x}$ and $c_{y}$ are mutually prime integers, $\mathbf{e}_{x(y)}$ are the unit vectors, while $d=|\mathbf{d}|$ is the length of the unit cell. Since ribbon edges are not flat, we define the effective ribbon width as the total square of hexagonal plaquettes inside the unit cell divided by the unit-cell width $d$.
TABLE I. The effective masses along the main axis for the electron and the hole, where $m_{0}$ is the free-electron mass.

\begin{tabular}{lcc}
\hline \hline & $x-\mathrm{ZZ}$ & $y$-AC \\
\hline$m_{e}^{*}$ (the electron) & $1.1547 m_{0}$ & $0.1951 m_{0}$ \\
& $0.848 m_{0}{ }^{\mathrm{a}}$ & $0.167 m_{0}{ }^{\mathrm{a}}$ \\
$m_{h}^{*}$ (the hole) & $3.2279 m_{0}$ & $0.1651 m_{0}$ \\
& $1.142 m_{0}{ }^{\mathrm{a}}$ & $0.184 m_{0}{ }^{\mathrm{a}}$ \\
\hline
\end{tabular}

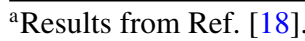

It is straightforward to show that the effective masses of electrons and holes in the vicinity of the $\Gamma$ point along the $x^{\prime}$ and $y^{\prime}$ axes, which are directed along and perpendicular to the ribbon edge, are, respectively, given by

$$
\begin{aligned}
\frac{1}{m_{e(h), x^{\prime}}^{*}} & =\frac{1}{m_{e(h), x}^{*}} \cos ^{2} \varphi+\frac{1}{m_{e(h), y}^{*}} \sin ^{2} \varphi \\
& = \pm\left.\frac{m_{0}}{\hbar^{2}} \lim _{k_{x^{\prime}} \rightarrow 0} \frac{d^{2} E_{e(h)}}{d k_{x^{\prime}}^{2}}\right|_{k_{y^{\prime}}=0}, \\
\frac{1}{m_{e(h), y^{\prime}}^{*}} & =\frac{1}{m_{e(h), x}^{*}} \sin ^{2} \varphi+\frac{1}{m_{e(h), y}^{*}} \cos ^{2} \varphi \\
& = \pm\left.\frac{m_{0}}{\hbar^{2}} \lim _{k_{y^{\prime}} \rightarrow 0} \frac{d^{2} E_{e(h)}}{d k_{y^{\prime}}^{2}}\right|_{k_{x^{\prime}}=0},
\end{aligned}
$$

where $E_{e(h)}$ denote the electron (hole) dispersion relation derived in the Appendix. Here $\varphi=\arctan \left(c_{y} a_{y} / c_{x} a_{x}\right)$ is the angle of rotation with respect to the $(x, y)$ coordinate system shown in Fig. 1. To write the Hamiltonian in the rotated frame, we replace $k_{x} \rightarrow k_{x^{\prime}} \cos \varphi-k_{y^{\prime}} \sin \varphi$ and $k_{y} \rightarrow k_{x^{\prime}} \sin \varphi+$ $k_{y^{\prime}} \cos \varphi$ in Eqs. (2) and (3). For infinitely long nanoribbons with arbitrary edges, the Hamiltonian should not depend on $x^{\prime}$, therefore $\phi_{c(v)}\left(x^{\prime}, y^{\prime}\right)=\phi_{c(v)}\left(y^{\prime}\right) e^{i k_{x^{\prime}} x^{\prime}}$. For convenience, we set $y^{\prime}=0$ in the middle of the ribbon.

We include the magnetic field perpendicular to the structure $\left(\mathbf{B}=B \mathbf{e}_{z}\right)$, which implies that in the continuum model, $k_{x^{\prime}} \rightarrow k_{x^{\prime}}-y^{\prime} / l_{B}^{2}$, where we adopt the Landau gauge $\mathbf{A}=$ $\left(-B y^{\prime}, 0,0\right)$, with $l_{B}=\sqrt{\hbar / e B}$ being the magnetic length and operators $k_{x^{\prime}}=-i \partial / \partial x^{\prime}$ and $k_{y^{\prime}}=-i \partial / \partial y^{\prime}$. Using Eqs. (6) and (7) and after some elaborate algebra, we obtain a set of decoupled differential equations for the conduction and valence bands,

$$
\begin{aligned}
& \frac{\hbar^{2}}{2 m_{e(h), y^{\prime}}^{*}}\left[-i \frac{\partial}{\partial \tilde{y}}-\tilde{y}\left(\frac{m_{e(h), y^{\prime}}^{*}}{m_{e(h), x^{\prime}}^{*}}-1\right) \frac{\tan 2 \varphi}{2 l_{B}^{2}}\right]^{2} \phi_{c(v)}(\tilde{y}) \\
& +\frac{1}{2} m_{e(h), y^{\prime}}^{*} \omega_{e(h)}^{2} \tilde{y}^{2} \phi_{c(v)}(\tilde{y})=\Delta E_{c(v)} \phi_{c(v)}(\tilde{y}),
\end{aligned}
$$

where the subscripts $c$ and $e$ are used for the conduction band, and $v$ and $h$ denote the valence band. Here, $\tilde{y}=y^{\prime}-k_{x}^{\prime} l_{B}^{2}$ is the shifted $y^{\prime}$ coordinate, $\omega_{e(h)}=e B / \sqrt{m_{e(h), x}^{*} m_{e(h), y}^{*}}$ are the cyclotron frequencies for the electron (hole) in bulk phosphorene, and $\Delta E_{c(v)}= \pm\left(E_{c(v)}-E_{c(v) 0}\right)$ are eigenenergies measured from the bottom (top) of the conduction (valence) -band edges. One may notice that the above equation does not include the term related to the band offset at the edges of the ribbon. This is because we want to analyze the conditions under which LLs exist in a ribbon. Namely, we insist that either the ribbon is 
sufficiently wide or the magnetic field is sufficiently strong, so that particle localization is governed by the effective parabolic potential $V_{e(h)}\left(k_{x^{\prime}}, y^{\prime}, B\right)=\frac{1}{2} m_{e(h), y^{\prime}} \omega_{e(h)}^{2}\left(y^{\prime}-l_{B}^{2} k_{x}^{\prime}\right)^{2}$, which is given in Eq. (8), rather than by the ribbon edges.

We seek the eigenvector components in the form

$$
\begin{aligned}
\phi_{c(v)}(\tilde{y})= & \exp \left(-\frac{m_{e(h), y^{\prime}}^{*} \omega_{e(h)}}{\hbar} \frac{\tilde{y}^{2}}{2}\right) \\
& \times \exp \left[i\left(\frac{m_{e(h), y^{\prime}}^{*}}{m_{e(h), x^{\prime}}^{*}}-1\right) \frac{\tan 2 \varphi}{2 l_{B}^{2}} \frac{\tilde{y}^{2}}{2}\right] f_{c(v)}(\tilde{y}),
\end{aligned}
$$

which leads to the differential equation

$$
f_{c(v)}^{\prime \prime}(\xi)-2 \xi f_{c(h)}^{\prime}(\xi)+\left(\varepsilon_{c(v)}-1\right) f_{c(v)}(\xi)=0,
$$

where $\xi=\xi_{e(h)}=\sqrt{m_{e(h), y^{\prime}}^{*} \cdot \omega_{e(h)} / \hbar} \cdot \tilde{y}$ is the dimensionless coordinate and $\varepsilon_{e(h)}=2 \Delta E_{c(v)} / \hbar \omega_{e(h)}$ is the dimensionless energy. Finally, to get eigenvalues denoted by the integer quantum number, we impose the condition $\varepsilon_{c(v)}-1=2 n_{e(h)}$. Thus, the solutions of Eq. (10) are Hermite polynomials,

$$
f_{c(v)}(\xi)=C_{n} H_{n}(\xi)=C_{n}(-1)^{n} e^{\xi^{2}} \frac{d^{n} e^{-\xi^{2}}}{d \xi^{n}},
$$

where the principal quantum number $n \triangleq n_{e(h)}=\{0,1,2, \ldots\}$ is the LL number, the normalization constant is $C_{n}=$ $\sqrt[4]{m_{e(h), y^{\prime}}^{*} \cdot \omega_{e(h)}} / \sqrt{n ! 2^{n} \pi^{1 / 2} \hbar^{1 / 2}}$, and the eigenvalues of the LLs follow the quantization of a one-dimensional quantum harmonic oscillator $(\mathrm{QHO})$,

$$
\begin{aligned}
& E_{c}^{\mathrm{LL}}=E_{c 0}+\hbar \omega_{e}\left(n_{e}+\frac{1}{2}\right), \\
& E_{v}^{\mathrm{LL}}=E_{v 0}-\hbar \omega_{h}\left(n_{h}+\frac{1}{2}\right) .
\end{aligned}
$$

It is obvious that the separation between adjacent energy levels $\left(\left|\Delta n_{e(h)}\right|=1\right)$ is approximately $\Delta E_{c(v)}=\hbar \omega_{e(h)}$. This energy difference is independent of edges and is equal to the one found in a single-layer phosphorous sheet [18,19].

It should be pointed out that the exact treatment of LLs in bulk phosphorene shows that the off-diagonal terms, which account for the conduction- and valence-band coupling, are similar in form to the Rashba (Dresselhaus) spin-orbit interaction in conventional semiconductors [23]. However, these terms do not contribute significantly to the spectra of the lowest energy states, and the spatial density distribution corresponding to the first few LLs is found to have an elliptical shape [18]. In a quasiclassical picture, we might infer that, when the magnetic field is turned on perpendicular to the bulk phosphorene sheet, electrons and holes undergo elliptical cyclotron orbits.

Let us now consider the impact of a perpendicular magnetic field. When the magnetic field is turned on, the particle is essentially confined in the transverse direction by a restricted effective parabolic potential $V_{e(h)}\left(k_{x^{\prime}}, y^{\prime}, B\right)$. One may notice that an increase (decrease) of $k_{x^{\prime}}$ shifts the effective parabolic potential toward the upper (lower) ribbon edge for both the electron and the hole. Therefore, states occupying positive and negative momenta reside on opposite sides of the ribbon. Also, the magnetic field does not separate oppositely charged particles in the transverse direction, and the electron and the hole will have similar localization in space even when the magnetic field is turned on. Furthermore, the effective potential is proportional to the transverse effective mass $m_{e(h), y^{\prime}}^{*}$. Thus, the confinement will be stronger in ribbons with higher effective mass in the transverse direction.

For relatively low values of $B$, the influence of the parabolic potential is small and the wave function is essentially determined by edges of the ribbon, i.e., the potential in the transverse direction resembles an infinite potential well. However, when the eigenvalue energy of the $n_{e(h)}$ th electron (hole) state in bulk phosphorene is lower (higher) than the effective potential at the ribbon edge closer to the potential extrema, i.e., when

$$
\hbar \omega_{e(h)}\left(n_{e(h)}+\frac{1}{2}\right)<\frac{1}{2} m_{e(h), y^{\prime}} \omega_{e(h)}^{2}\left(\frac{W}{2}-l_{B}^{2}\left|k_{x^{\prime}}\right|\right)^{2},
$$

the confinement along the $y^{\prime}$ direction is dominantly QHOlike. We should note that this criterion is not so rigid. Namely, the effective potential at the closer edge should be sufficiently larger than the QHO eigenenergy so that the corresponding eigenfunction decreases sufficiently before it reaches the ribbon edge. More comprehensive criteria are established in Ref. [24], but since we have already made a few approximations, it would not improve the analytical results significantly.

The smallest value of the magnetic field for LL formation in the $n$th electron (hole) state is found when $k_{x^{\prime}}=0$, and $<$ in Eq. (14) is replaced by an equality sign:

$$
B_{n_{e(h)}^{\min }}=\frac{4 \hbar\left(2 n_{e(h)}+1\right)}{e W^{2}} \frac{\sqrt{m_{e(h), x}^{*} m_{e(h), y}^{*}}}{m_{e(h), y^{\prime}}^{*}} .
$$

Note that the minimal field $B_{n_{e(h)}}^{\min }$ is proportional to the number of LLs. In the above equation, only the transverse effective mass $m_{e(h), y^{\prime}}^{*}$ depends on the edge orientation, and $B_{n_{e(h)}}^{\min }$ decreases monotonically with it. In phosphorene, the largest value of the effective mass is in the zigzag direction, while the smallest one is in the armchair direction. Therefore, the magnetic field required for the formation of the LLs is the smallest in the case of $\mathrm{AC}$ ribbons and the largest for $\mathrm{ZZ}$ ribbons. A similar conclusion could be drawn intuitively from the analysis of the effective potential, based on the argument related to the confinement strength.

Furthermore, when $B>B_{n_{e(h)}}^{\min }$ we deduce from inequality (14) that in the range $k_{x^{\prime}} \in\left(-k_{n_{e(h)}, x^{\prime}}^{\mathrm{FB}}, k_{n_{e(h)}, x^{\prime}}^{\mathrm{FB}}\right)$, eigenvalues become independent of $k_{x^{\prime}}$, where

$$
k_{n_{e(h)}, x^{\prime}}^{\mathrm{FB}}=\frac{1}{l_{B}}\left[\frac{W}{2 l_{B}}-\sqrt{2 n_{e(h)}+1}\left(\frac{\sqrt{m_{e(h), x}^{*} m_{e(h), y}^{*}}}{m_{e(h), y^{\prime}}^{*}}\right)^{\frac{1}{2}}\right]
$$

is the flatband boundary wave number. Namely, the band structure of states that satisfy the condition (14) should appear flat. It is straightforward to show that the flatband boundary wave number increases with transverse mass. Therefore, the flatband range is the widest for a ribbon with $\mathrm{AC}$ edges and the smallest for a $\mathrm{ZZ}$ ribbon. Furthermore, the width of the flatband decreases with increasing LL number, which is due 
to the fact that higher states extend more along the width of the ribbon, so the condition that the wave function "touches" the ribbon edge given by inequality (14) is satisfied for smaller values of the longitudinal momentum.

Let us also briefly discuss the influence of an in-plane electric field $E_{y^{\prime}}$ on the LLs. Such an external electric field modifies the on-site energy. Therefore, in the continuum model we add the potential $V^{\operatorname{ext}}\left(E_{y}^{\prime}, y^{\prime}\right)=e E_{y^{\prime}} y^{\prime}=$ $e E_{y^{\prime}}\left(\tilde{y}+l_{B}^{2} k_{x^{\prime}}\right)$ to the diagonal terms. It is straightforward to show that the differential equations (8) are modified so that the effective potential becomes $V_{e(h)}\left(k_{x^{\prime}}, y^{\prime}, B\right)=$ $\frac{1}{2} m_{e(h), y^{\prime}} \omega_{e(h)}^{2}\left[y^{\prime}-l_{B}^{2} k_{x}^{\prime} \pm e E_{y^{\prime}} /\left(m_{e(h), y^{\prime}} \omega_{e(h)}^{2}\right)\right]^{2}$, while two terms are added to the right side of the equations, $\Delta E_{c(v)} \rightarrow$ $\Delta E_{c(v)}+e E_{y^{\prime}} k_{x^{\prime}} l_{B}^{2} \mp e^{2} E_{y^{\prime}}^{2} /\left(2 m_{e(h), y^{\prime}} \omega_{e(h)}^{2}\right)$. Finally, we obtain similar solutions for the eigenfunctions as for the case when only the magnetic field is applied. As expected, the wave functions are shifted along the ribbons width in the direction of transverse electric field for holes, and in the opposite direction for electrons. Also, eigenenergies of LLs are modified,

$$
E_{c(v)}=E_{c(v)}^{\mathrm{LL}}\left(E_{y^{\prime}}=0\right) \mp \frac{e^{2} E_{y^{\prime}}^{2}}{2 m_{e(h), y^{\prime}} \omega_{e(h)}^{2}}+e E_{y^{\prime}} k_{x^{\prime}} l_{B}^{2} .
$$

We infer that the energy gap between the states in the conduction and valence bands with the same $k_{x^{\prime}}$ is reduced by $e^{2} E_{y^{\prime}}^{2} /\left(m_{e(h), y^{\prime}} \omega_{e(h)}^{2}\right)$, while formerly flatbands adopt linear dispersion $\left(\sim e E_{y^{\prime}} k_{x^{\prime}} l_{B}^{2}\right)$ turning the band gap to indirect. This behavior is expected since $B$ leads to a shift of states with positive (negative) momenta to the upper (lower) side of the ribbon, and therefore electrons and holes experience opposite potential shifts. Moreover, the wave numbers that determine the boundaries of these linear regions can be found from (14) when $k_{x^{\prime}}$ is substituted by $k_{x^{\prime}} \mp \Delta k\left(E_{y^{\prime}}, B\right)$, where $\Delta k\left(E_{y^{\prime}}, B\right)=$ $e E_{y^{\prime}} /\left(m_{e(h), y^{\prime}} \omega_{e(h)}^{2} l_{B}^{2}\right)=\left(E_{y^{\prime}} / B \hbar\right) m_{e(h), x} m_{e(h), y} / m_{e(h), y^{\prime}}$. One may note that these spectral shifts depend linearly on $E_{y^{\prime}}$. Moreover, based on the argument regarding the value of the effective mass in a certain direction, it is easy to conclude that these shifts are smallest in a ribbon with $\mathrm{AC}$ edges and are largest for the one with $\mathrm{ZZ}$ edges. Consequently, the band dispersions become tilted when $E_{y^{\prime}}$ is applied.

The presented continuum model does not account for the edge states. The presence and the origin of these states in ribbons with various edges were discussed in detail in Refs. [15,16]. The recently proposed continuum model with proper boundary conditions results in adequately modeled edge states for ZZ ribbons [25]. In a former approach [16], the edge states are treated as quasiflat bands and are determined as the zero-energy states in the anisotropic honeycomb lattice model.

We note that for chosen directions of the magnetic and electric fields, the wave functions are symmetric along the $x^{\prime}$ direction of the ribbon's unit cell. Therefore, the effective translation vector that is twice as short $(\mathbf{d} / 2)$ can be introduced. The same argument is used for the derivation of the two-band Hamiltonian. As a consequence, the first Brillouin zone (FBZ) becomes twice as wide and the energy spectrum unfolds. The calculation times become more than twice as short.

In addition to the calculation of electron states, we model electron transport by using the formalism of a nonequilibrium Green's functions [26]. The structure is assumed to be connected to the environment by semi-infinite leads, and conductance is determined for the case of linear response using the Fisher-Lee relation [27]

$$
\sigma(E)=\frac{e^{2}}{h} \operatorname{Tr}\left(\Gamma_{L}(E) G_{D}^{\dagger}(E) \Gamma_{R}(E) G_{D}(E)\right) .
$$

Here, $G_{D}$ denotes the device Green's function, which is given by

$$
G_{D}(E)=\left[E-H_{D}-\Sigma_{L}(E)-\Sigma_{R}(E)\right]^{-1},
$$

where $\Sigma_{L(R)}$ are the self-energies:

$$
\Sigma_{L(R)}(E)=H_{L(R) D}^{\dagger} g_{L(R)} H_{L(R) D},
$$

and $g_{L(R)}(E)=\left(E-H_{L(R)}\right)^{-1}$ are the Green's functions of the left (right) leads. Here, $H_{D}$ is the device Hamiltonian, $H_{L(R)}$ describe hopping in the left (right) lead, $H_{L(R) D}$ describe hopping from the device to the left (right) lead, and $\Gamma_{L(R)}$ is the broadening matrix,

$$
\Gamma_{L(R)}(E)=i\left(\Sigma_{L(R)}(E)-\Sigma_{L(R)}^{\dagger}(E)\right) .
$$

A detailed description of the transport model as well as procedures for its implementation are explained in Refs. [29,30], and references cited therein.

\section{NARROW NANORIBBONS IN COMBINED FIELDS}

Our goal is to compare nanoribbons with equal width having different edges. Therefore, we chose the number of dimers along the ribbon cross section to be $61,38,46$, and 74 , so we have approximately 10 -nm-wide nanoribbons with AC, sZZ, ZZ, and sAC edges, respectively. And we chose the value of a perpendicular applied magnetic field $B=500 \mathrm{~T}$, such that Landau level formation can be seen. Notice that the critical field according to Eq. (15) is inversely proportional to the square of the ribbon width. Furthermore, for 10-nm-wide nanoribbons, energy levels are substantially separated, such that LLs are visualized with more details. Yet, the results do not qualitatively change if the nanoribbon width is increased, which will be demonstrated later for an order of magnitude wider nanoribbons. The eigenenergies are calculated numerically using the tight-binding Hamiltonian given by Eq. (1).

In Fig. 2, we show the band structures of $\mathrm{AC}$ nanoribbons for the following cases: (a) in the absence of fields, (b) with applied magnetic field only, and (c) in the presence of both $B$ and $E_{y^{\prime}}$. In the absence of fields these nanoribbons are insulating [15], and they host a series of parabolic bands, which are effectively "sampled" from the band structure of bulk phosphorene, as might be inferred from Fig. 2(a). When the magnetic field is turned on, LLs are formed and flatbands appear, as shown in Fig. 2(b). Quasiclassically, for states away from the edges, the magnetic field enforces closed elliptical cyclotron orbits. These states in turn quantize into LLs and form the flat parts of the bands displayed in Fig. 2(b). Note that these segments get narrower at higher energies and lower magnetic fields; the reason for this is that the cyclotron radius enlarges, and therefore fewer orbits are uninterrupted by the edges. 

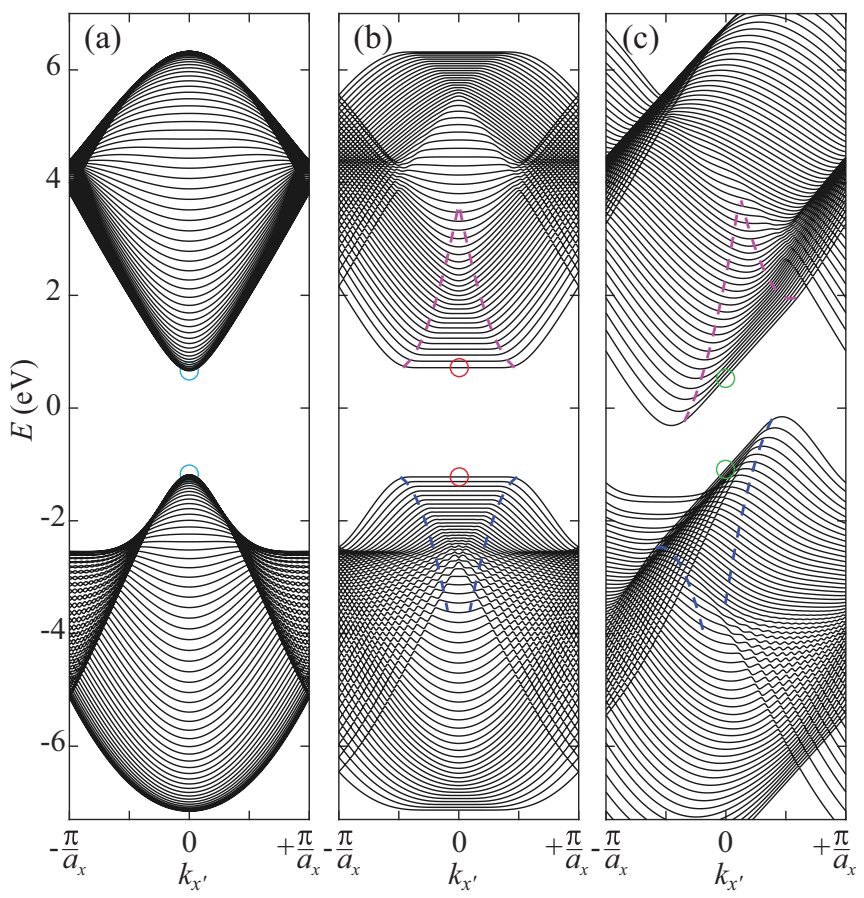

FIG. 2. The band structure of $N_{\mathrm{AC}}=61$ nanoribbon for (a) $B=0$ and $E_{y^{\prime}}=0$, (b) $B=500 \mathrm{~T}$ and $E_{y^{\prime}}=0$, and (c) $B=500 \mathrm{~T}$ and $E_{y^{\prime}}=200 \mathrm{mV} / \mathrm{nm}$. Dashed magenta (blue) lines in the conduction (valence) band denote theoretically calculated edges of the flat and linear bands in (b) and (c), respectively.

As we discussed in Sec. II, due to the large effective mass in the transverse $\mathrm{ZZ}$ direction, confinement is strong, and LLs might be found almost as soon as $B$ is turned on. Therefore, a large number of LLs are supported by nanoribbons with AC edges. Dashed magenta and blue curves denote theoretically calculated edges of the flatbands in the conduction and valence band, respectively. There is good agreement between these edges and our numerical results. One of the reasons for certain small differences is explained in Sec. II, and is related to the flexible condition for these edges, given in Eq. (14). The other reasons are the finite width of the ribbon and interband coupling [18], due to which the spacing between LLs decreases as we move from the top (bottom) of the valence (conduction) band. Therefore, $\Delta E_{c(v)}^{\mathrm{LL}}$ are overestimated, and slopes of the theoretically calculated edges are higher than the actual ones. Furthermore, we must bear in mind that the continuum approximation is valid in a relatively narrow range of momenta around the $\Gamma$ point. Consequently, the discrepancy between the analytical and numerical results is larger for the AC ribbon.

When an electric field $E_{y^{\prime}}$ is applied, the flatbands become linearly dependent on $B$ and appear as tilted. As predicted by theory, due to the linear term the band gap switches to an indirect one as soon as the in-plane electric field is applied. For the value of the electric field $E_{y^{\prime}}=200 \mathrm{mV} / \mathrm{nm}$, the band gap closes, as might be observed from Fig. 2(c). Furthermore, shifts of these linear segments in momentum space seem to be almost equal for the conduction and valence band. Earlier, we analyzed the spectral shift with respect to the effective
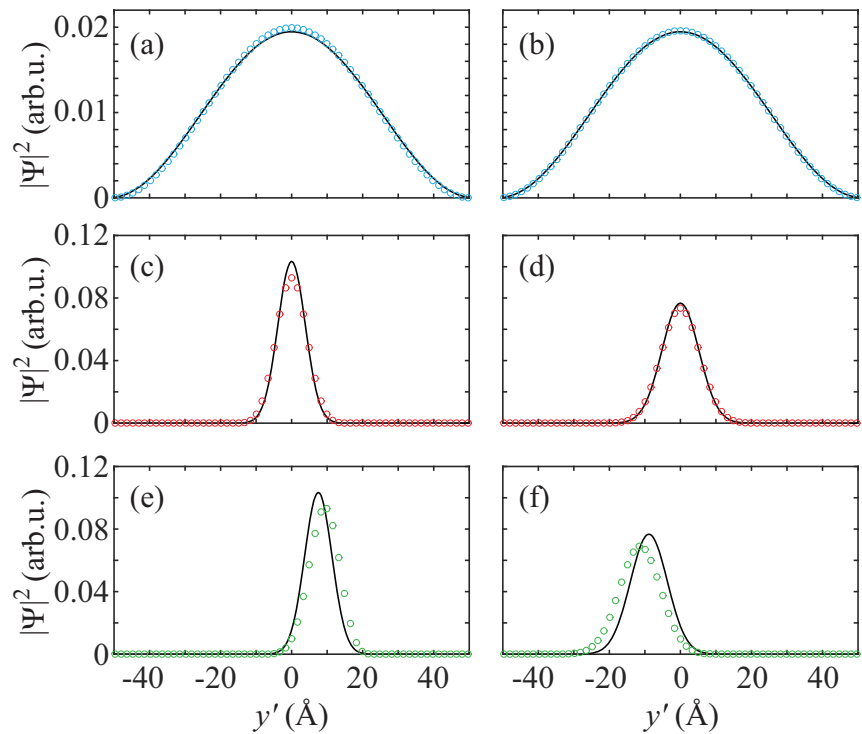

FIG. 3. Left (right) column depicts the probability density of states in the $N_{\mathrm{AC}}=61$ nanoribbon in the top of the valence (bottom of the conduction) band at $k_{x^{\prime}}=0$ when (a),(b) $B=0$ and $E_{y^{\prime}}=0$ (first row), (c),(d) $B=500 \mathrm{~T}$ and $E_{y^{\prime}}=0$ (second row), and (e),(f) $B=500 \mathrm{~T}$ and $E_{y^{\prime}}=100 \mathrm{mV} / \mathrm{nm}$ (third row). The solid curves and the symbols show $|\Psi|^{2}$ found from the continuum and the tight-binding model, respectively.

masses and found that $\Delta k \sim m_{e(h), x} m_{e(h), y} / m_{e(h), y^{\prime}}$. In the case of AC ribbons, $\Delta k^{\mathrm{AC}} \sim m_{e(h), y}$, where $m_{e, y}=0.195 m_{0}$ and $m_{h, y}=0.165 m_{0}$, thus the spectral shifts have similar values for electrons and holes. We note that only for AC ribbons is the shift slightly larger for electrons than for holes. For all the other ribbons discussed in the paper, the mass-dependent part of the shift for the holes is approximately 1.5-3 times larger than for the electrons. Therefore, we expect that for ribbons other than of the AC type, the holes are much more sensitive to the in-plane field than the electrons.

In Fig. 3 we show the real-space probability density of states at the top (bottom) of the valence (conduction) band for $k_{x^{\prime}}=0$. The states are marked with correspondingly colored circles in Fig. 2. Numerical results are compared to analytical solutions. To compare probability densities found by the tight-binding model to those determined by means of the continuum approximation, we divide the probability for occupying a certain state by the distance between the adjacent atoms in each sublattice, i.e., $\Delta y_{\mathrm{AC}}^{\prime}=a_{r} / 2$ for $\mathrm{AC}$ and $\Delta y^{\prime}=\frac{c_{r}}{2} \cos \varphi=\frac{\left|a_{t}\right|}{d} \frac{a_{r} c_{r}}{2}$ for all the others. When magnetic and electric fields are not present, confinement along the transverse direction is as in an infinite potential well. Wave functions that are obtained numerically (denoted by open circles in Fig. 3) are compared to the theoretical expression $\Psi_{0}\left(y^{\prime}, k_{x^{\prime}}=0\right)=\sqrt{\frac{2}{W}} \cos \left(\frac{\pi}{W} y^{\prime}\right)$ displayed by solid lines in Figs. 3(a) and 3(b), for the hole and the electron, respectively. We found that the wave function is spread along the ribbons with similar distribution for both the electron and the hole, as predicted by the theoretical expression. According to Eq. (9) for the case when both magnetic and electric fields are applied, 


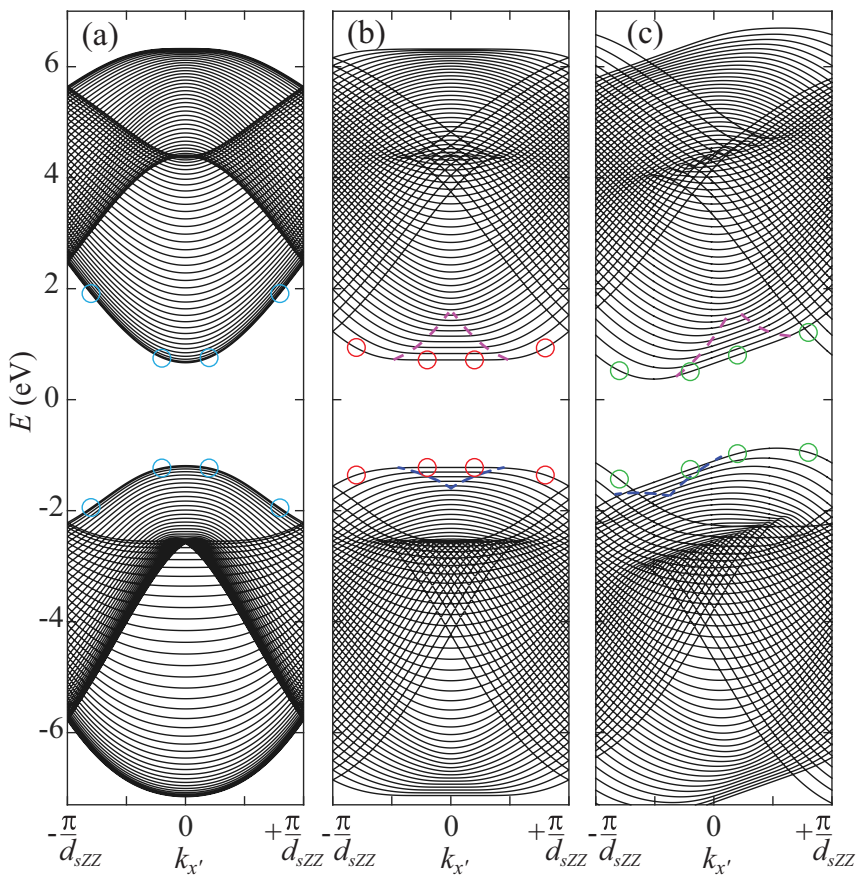

FIG. 4. The band structure of $N_{\mathrm{sZZ}}=38$ nanoribbon for (a) $B=0$ and $E_{y^{\prime}}=0$, (b) $B=500 \mathrm{~T}$ and $E_{y^{\prime}}=0$, and (c) $B=500 \mathrm{~T}$ and $E_{y^{\prime}}=100 \mathrm{mV} / \mathrm{nm}$.

the ground-state wave function at $k_{x^{\prime}}=0$ reads

$$
\begin{aligned}
\left|\psi_{0, e(h)}^{\mathrm{QHO}}\left(y^{\prime}, k_{x^{\prime}}=0\right)\right|= & \left(\frac{m_{e(h), y^{\prime}} \omega_{e(h)}}{\pi \hbar}\right)^{\frac{1}{4}} \exp \left[-\frac{m_{e(h), y^{\prime}} \omega_{e(h)}}{2 \hbar}\right. \\
& \left.\times\left(y^{\prime} \pm \frac{e E_{y^{\prime}}}{m_{e(h), y^{\prime}} \omega_{e(h)}^{2}}\right)^{2}\right]
\end{aligned}
$$

When only the magnetic field is present, we found excellent agreement between our numerical results and the theoretically predicted Gaussian function from our simplified model. Namely, almost perfect bell-shaped functions are formed in the middle of the ribbon, as may be inferred from Figs. 3(c) and 3(d) for the hole and electron, respectively. Good agreement is also found in the presence of an electric field, as shown in Figs. 3(d) and 3(e). The wave functions remain Gaussian-like but shift toward the upper (lower) edge for the hole (electron).

Next, we display the evolution of the band structure of sZZ nanoribbons in magnetic and electric fields in Fig. 4. In the absence of the fields, similarly for AC, these ribbons are insulating, as inferred from Fig. 4(a). When sufficiently large $B$ is applied, LLs are formed in the middle of the FBZ, i.e., the parabolic bands evolve, and they start featuring dispersionless segments, as we observed in Fig. 4(b). By inspection of Fig. 4(b), we note that there are fewer flat segments that are somewhat narrower than in the case of the AC ribbon with approximately the same width.

To elucidate these effects, in Fig. 5 we plot the real-space probability density of the ground states marked with correspondingly colored circles in Fig. 4, where solid and dashed curves depict $|\Psi|^{2}$ on opposite sublattices. Both sublattices exhibit the same functional variation of $|\Psi|^{2}$, with a slight
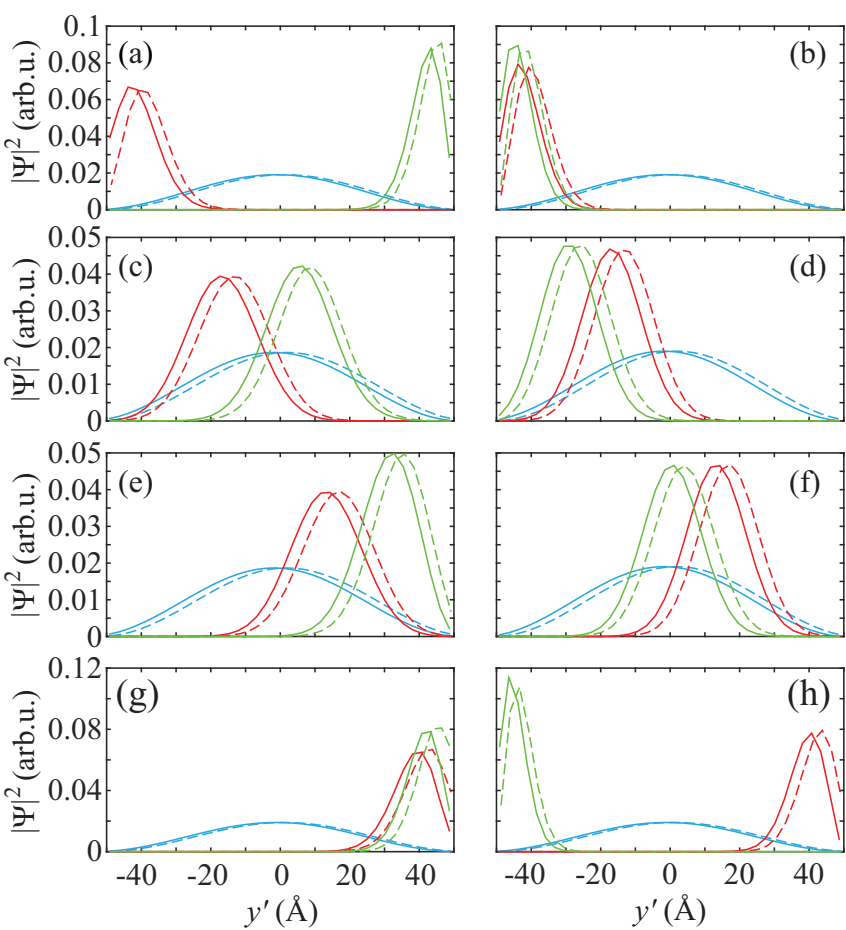

FIG. 5. The left (right) column depicts the probability density of the ground states of the $N_{\mathrm{szz}}=38$ nanoribbon in the top of the valence (bottom of the conduction) band at $k_{x^{\prime}}=-0.8 \pi / d_{\text {sZZ }}$ (first row), $k_{x^{\prime}}=-0.2 \pi / d_{\mathrm{szZ}}$ (second row), $k_{x^{\prime}}=0.2 \pi / d_{\mathrm{szZ}}$ (third row), and $k_{x^{\prime}}=0.8 \pi / d_{\text {szz }}$ (fourth row). The light blue lines correspond to $B=0$ and $E_{y^{\prime}}=0$, red lines correspond to $B=500 \mathrm{~T}$ and $E_{y^{\prime}}=0$, while green lines correspond to $B=500 \mathrm{~T}$ and $E_{y^{\prime}}=100 \mathrm{mV} / \mathrm{nm}$. The solid and dashed lines show $|\Psi|^{2}$ of the opposite sublattices.

lateral offset, which is a general property independent of the applied fields. The left (right) panel column corresponds to the valence (conduction) band, while the first, second, third, and fourth rows in the panel correspond to $k_{x^{\prime}}=-0.8 \pi / d, k_{x^{\prime}}=$ $-0.2 \pi / d, k_{x^{\prime}}=0.2 \pi / d$, and $k_{x^{\prime}}=0.8 \pi / d$, respectively. In the absence of fields, the ground electron and hole states fully extend across the ribbon width, regardless of the $k_{x^{\prime}}$, as might be observed from the light blue curves in Figs. 5(a)-5(h). For nonzero $k_{x^{\prime}}$, energy increases as described by $\hbar^{2} k_{x^{\prime}}^{2} / 2 m_{e(h), x^{\prime}}$, while confinement along the ribbon width remains unchanged. The probability densities of the ground states corresponding to the dispersionless segments in the valence (conduction) band are shown by the red curves in Figs. 5(c)-5(f). We infer that these probability densities have an almost unchangeable Gaussian shape for any $k_{x^{\prime}}$ in the flatband range, as can be concluded by comparing the red curves in Figs. 5(c) and 5(e) for the valence band, as well as from Figs. 5(d) and 5(f) for the conduction band.

Next, we analyze the effects of an applied transverse electric field. By comparing the green curves in Figs. 5(b)-5(g) to the red ones, we found that the states are almost the same as in the case when only the magnetic field is applied. As expected, calculated wave functions in linear segments of the spectrum shown in Fig. 4(c) have a shape that is almost identical to the corresponding eigenfunctions in the flatbands. However, these states are shifted along the ribbon width due 

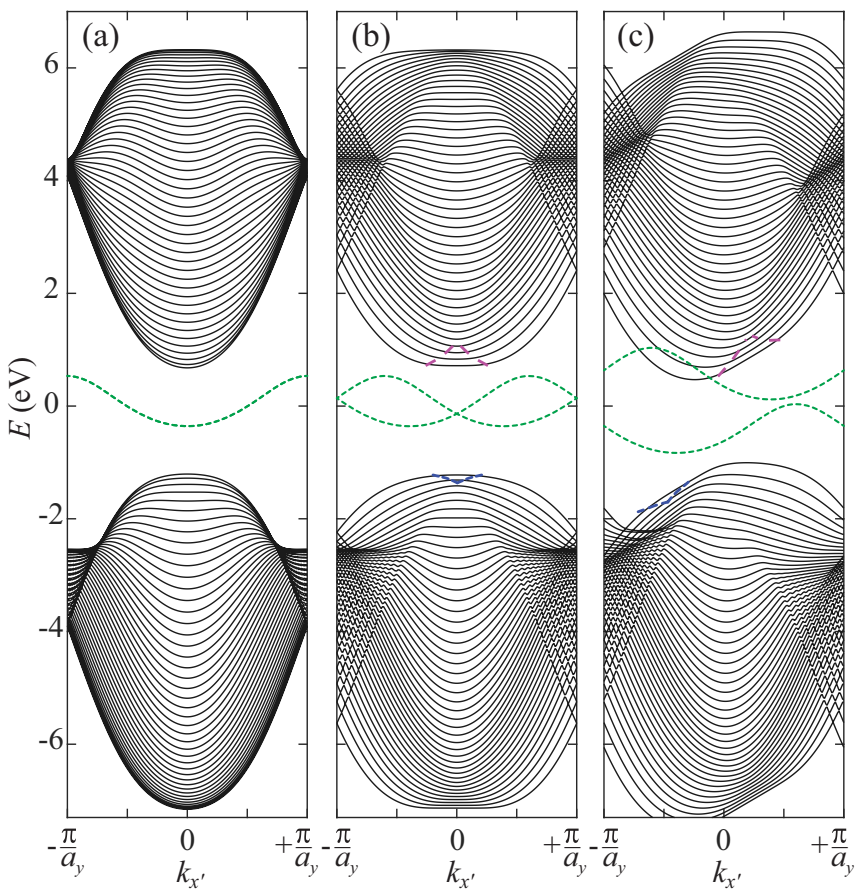

FIG. 6. The band structure of $N_{\mathrm{ZZ}}=46$ nanoribbon for (a) $B=0$ and $E_{y^{\prime}}=0$, (b) $B=500 \mathrm{~T}$ and $E_{y^{\prime}}=0$, and (c) $B=500 \mathrm{~T}$ and $E_{y^{\prime}}=100 \mathrm{mV} / \mathrm{nm}$. The edge states are shown by the dotted green line.

to the electric field. For negative values of the longitudinal momenta where the dispersion is linear [see linear segments limited by the dashed blue line in Fig. 4(c)], the hole wave function is localized around the center of the ribbon. Note that the valence-band ground state at $k_{x^{\prime}}=0.2 \pi / d$ is not in the linear region, as shown in Fig. 4(c). In fact, since $\Delta k_{h}^{\mathrm{sZZ}} / \Delta k_{e}^{\mathrm{sZZ}}=2.352$ is large, linear segments are much more shifted in the valence than in the conduction band, as displayed in Fig. 4(c). Therefore, the probability density displayed in Fig. 5(e) interferes with the upper ribbon edge, and it differs from the bell-shaped function shown in Fig. 5(c).

However, the most intriguing behavior occurs near the left zone edge, where crossing is found for the hole ground state. The crossing involves the states localized at the opposite edges of the ribbon [see Fig. 4(c)]. Therefore, the localization of the hole ground state can be abruptly changed by the electric field, as shown in Fig. 5(a). The same effect occurs in the conduction band, but near the upper edge of the FBZ [see Fig. 4(c)]. Due to the crossing, the electron localization switches to the opposite edge, as shown by the green curves in Fig. 5(h). Additionally, the position of the crossings can be tuned by a magnetic field.

The band structures of zigzag nanoribbons are shown in Fig. 6. As discussed in Sec. II, the interband coupling has a smaller influence on the motion along the $\Gamma-X$ direction, and the dispersion is almost perfectly parabolic in most of the bands [see Fig. 6(a)]. Since ZZ ribbons have the weakest confinement in the transverse direction even when high $B$ is applied, only a few LLs evolve [see the small regions encircled by dashed lines in Fig. 6(b)]. When the electric field is applied, bands become immediately tilted, and newly formed linear bands are shifted. By comparing these shifts in the conduction and

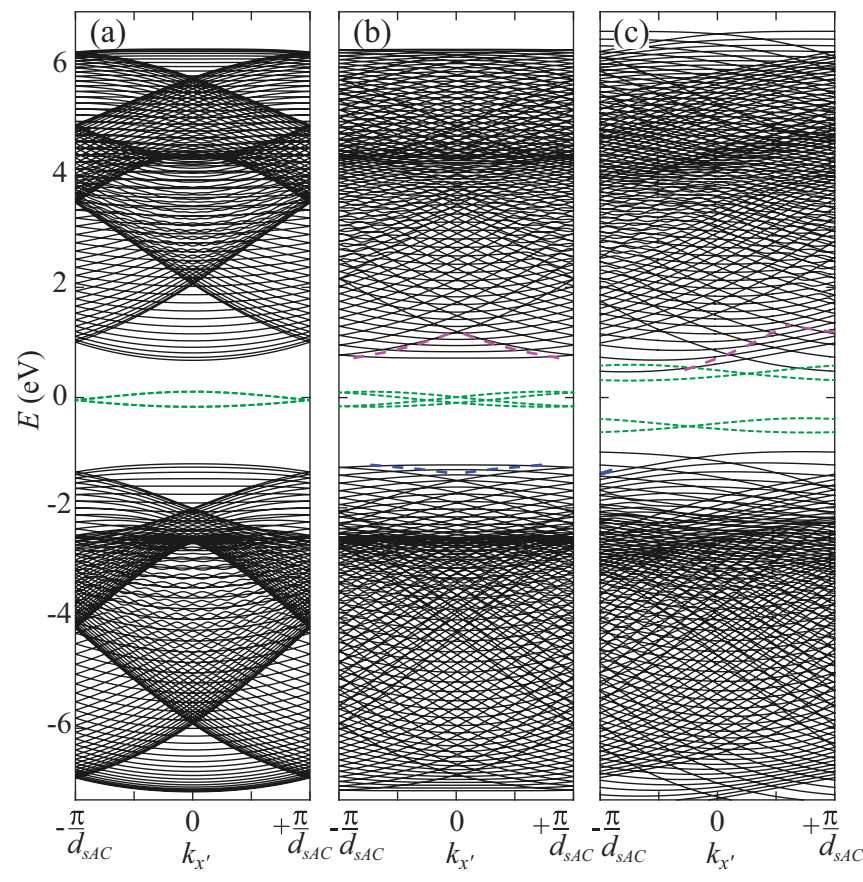

FIG. 7. The band structure of $N_{\mathrm{sAC}}=74$ nanoribbon for (a) $B=0$ and $E_{y^{\prime}}=0$, (b) $B=500 \mathrm{~T}$ and $E_{y^{\prime}}=0$, and (c) $B=500 \mathrm{~T}$ and $E_{y^{\prime}}=100 \mathrm{mV} / \mathrm{nm}$. The edge states are displayed by a dotted green line.

valence bands in Fig. 6(c), we realize that the hole is much more sensitive to the electric field, which is supported by the fact that the ratio $\Delta k_{h}^{\mathrm{ZZ}} / \Delta k_{e}^{\mathrm{ZZ}}=2.795$ for $\mathrm{ZZ}$ nanoribbons is the highest out of all considered ribbons.

In Fig. 7, we show the band structure of skewed armchair nanoribbons. Note that there are fewer flat segments in the spectrum of Fig. 7(b) corresponding to LLs forming around the middle of the ribbon than in the case of $\mathrm{AC}$ and $\mathrm{sZZ}$ ribbons. Also, these segments are much narrower, which can be explained by having in mind that the width of FBZ for $\mathrm{sAC}$ is $1.5-3$ times narrower than for the other ribbon types. Since $\Delta k_{h}^{\mathrm{sAC}} / \Delta k_{e}^{\mathrm{sAC}}=2.734$, linear segments are much more shifted in the valence than in the conduction band, and the dashed blue line delineating linear bands starts at the band edge, as shown in Fig. 7(c).

Finally, we investigate the behavior of edge states. These states are in the gap and have energies close to zero, as predicted by a simple model for anisotropic honeycomb lattices [16]. They do not undergo Landau quantization, as can be seen in Figs. 6(b) and 7(b), which is a consequence of their exponential localization near the edges [15]. Instead, each QFB is effectively shifted along the $k_{x^{\prime}}$ axis, but in opposite directions. This behavior was absent in the case of edge states in zigzag graphene ribbons, since the corresponding bands were not fully detached from the bulk bands [16], as is the case with edge states in phosphorene nanoribbons.

In Fig. 8, we take a closer look at QFBs for (a) $N_{\mathrm{ZZ}}=46$ and (b) $N_{\mathrm{sAC}}=74$ and a range of magnetic-field values. In the absence of a magnetic field, the bands are degenerate for both $\mathrm{ZZ}$ and sAC, as depicted by solid blue curves in Fig. 8 . It is clear that the QFBs are progressively shifted in opposite 

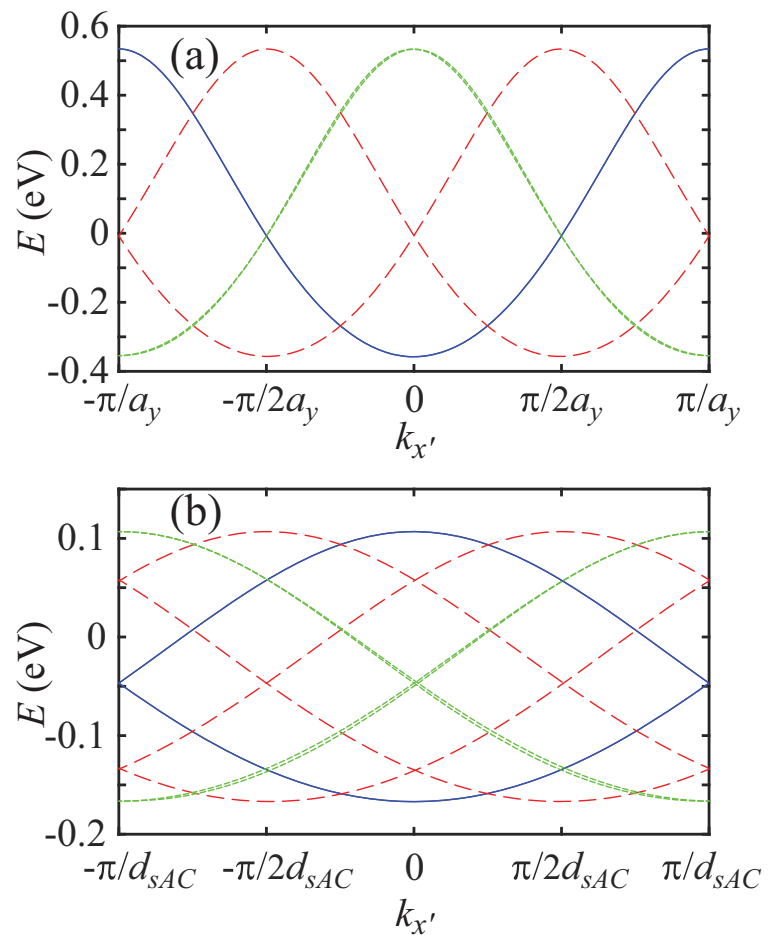

FIG. 8. The quasiflat bands for (a) $N_{\mathrm{ZZ}}=46$ and (b) $N_{\mathrm{sAC}}=74$ nanoribbon for $B=0$ (blue solid curves), $B=B_{\mathrm{cr}} / 2$ (red dashed curves), and $B=B_{\mathrm{cr}}$ (green dotted curves).

directions with increasing magnetic field (see the red dashed lines in Fig. 8). An explanation for this behavior is very similar to the one given in Ref. [28]: exponentially localized states "sample" the vector potential only in a small area where it is effectively constant, and therefore the effect of magnetic fields amounts only to a phase shift. Additionally, the two QFB states are localized at opposite edges (at $y^{\prime}= \pm W / 2$, for a ribbon of width $W)$, where the local vector potential $\mathbf{A}=B(-y, 0,0)$ has different signs, which explains the opposite shifts.

A quantitative description can be obtained by employing the principle of minimal coupling $k_{x^{\prime}} \rightarrow k_{x^{\prime}}+\frac{e}{\hbar} A_{x^{\prime}}$. In other words, the bands get shifted left and right by $\frac{e B W}{2 \hbar}$, depending on at which edge they are localized. We have confirmed that this is indeed a very good approximation for smaller fields. This also suggests that when $\pi / d=e B_{\mathrm{cr}} W / 2 \hbar$, where $d$ is the unit-cell length, QFBs get completely "out of phase," so that maxima and minima change places (see the green dotted curves in Fig. 8). This critical magnetic field is then given as $B_{\text {cr }}=2 \pi \hbar /(e d W)$, which in fact means that the magnetic flux through the unit cell $\left(\Phi_{\mathrm{cr}}=B_{\mathrm{cr}} d W\right)$ is equal to one flux quantum $\left(\Phi_{0}=h / e\right)$.

The exact expressions for the critical magnetic field read $B_{\mathrm{cr}}^{\mathrm{ZZ}}=\Phi_{0} /\left[\left(N_{\mathrm{ZZ}}-1\right) A_{\mathrm{hex}}\right]$ and $B_{\mathrm{cr}}^{\mathrm{sAC}}=$ $\Phi_{0} /\left[2\left(N_{\mathrm{SAC}}-2\right) A_{\text {hex }}\right]$ for $\mathrm{ZZ}$ and sAC ribbons, respectively. Here, $A_{\text {hex }}=a_{r} c_{r} / 2$ is the area of plaquette projection onto the $x, y$ plane. The green dotted curves in Fig. 8 show the band structure at $B_{\mathrm{cr}}$. However, these two bands are not degenerate and thus they are not exactly in opposition with respect to the solid blue curves. There are two reasons for this, both related to the fact that edge states have some finite spread toward the ribbon center. On the one hand, this means that the edge states effectively experience somewhat smaller ribbon widths (thus increasing true $B_{\mathrm{cr}}$ ). On the other hand, for larger magnetic fields the vector potential has a stronger spatial variation, so that the simple picture of phase shifts (assuming relatively constant $\mathbf{A}$ in the narrow space occupied by the edge state) gradually loses validity.

\section{WIDE NANORIBBONS IN COMBINED FIELDS}

In the previous section, we investigated magnetoelectronic properties of 10-nm-wide nanoribbons. To observe LLs in our calculations, it was necessary to adopt large values of $B$, which might stay beyond current experimental observations. Similarly, the value of the electric field that closes the band gap is high such that real samples could be damaged under these conditions. Experimental conditions could be approached by, e.g., assuming a larger nanoribbon width.

The band structure of 100-nm-wide AC, sZZ, ZZ, and sAC nanoribbons is shown in Fig. 9. The eigenenergies are calculated numerically using Eq. (1). The upper panel displays how LLs are formed in the presence of a magnetic field whose magnitude is $B=20 \mathrm{~T}$, whereas the lower panel shows a result when both a magnetic field $B=20 \mathrm{~T}$ and an electric

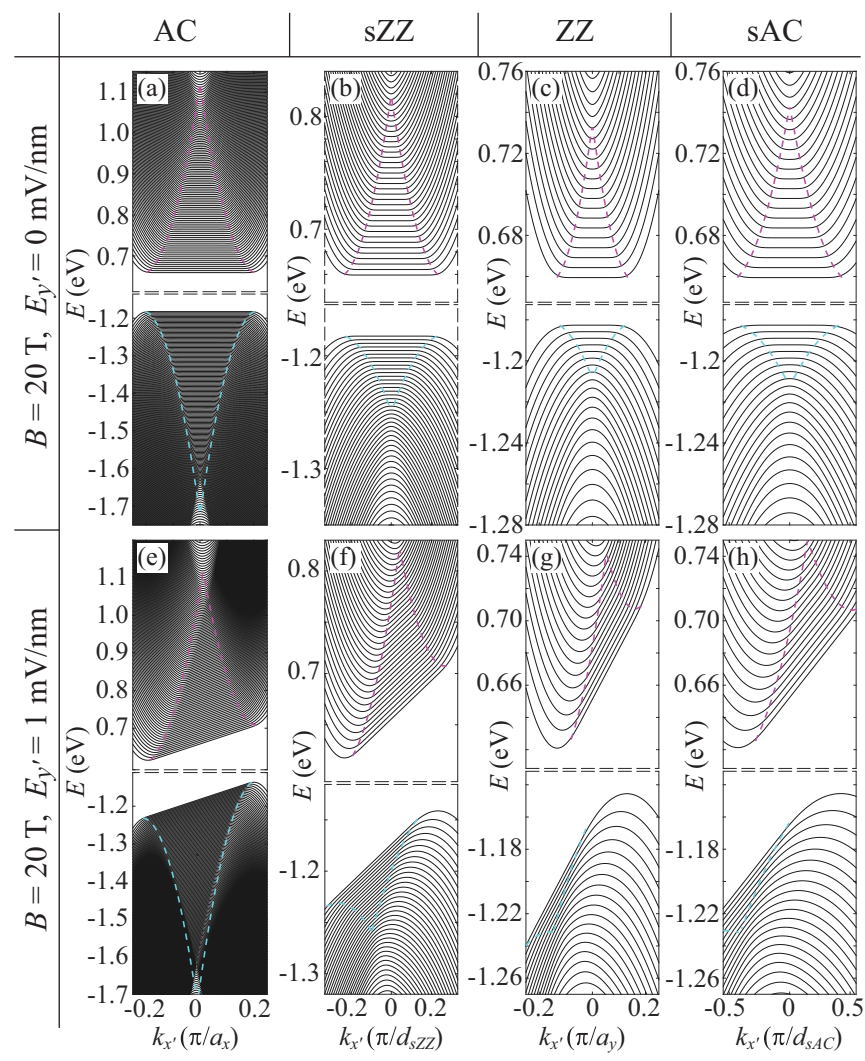

FIG. 9. Upper panel: the band structure of nanoribbons for $B=20 \mathrm{~T}$ : (a) $N_{\mathrm{AC}}=610$, (b) $N_{\mathrm{sZZ}}=380$, (c) $N_{\mathrm{ZZ}}=460$, and (d) $N_{\mathrm{sAC}}=740$. Lower panel: the same as in the upper panel but for $B=20 \mathrm{~T}$ and $E_{y^{\prime}}=1 \mathrm{mV} / \mathrm{nm}$ : (e) $N_{\mathrm{AC}}=610$, (f) $N_{\mathrm{sZZ}}=380$, (g) $N_{\mathrm{ZZ}}=460$, and (h) $N_{\mathrm{sAC}}=740$. Dashed magenta (cyan) curves denote borders of linear parts of the dispersion relations in the conduction (valence) band. 


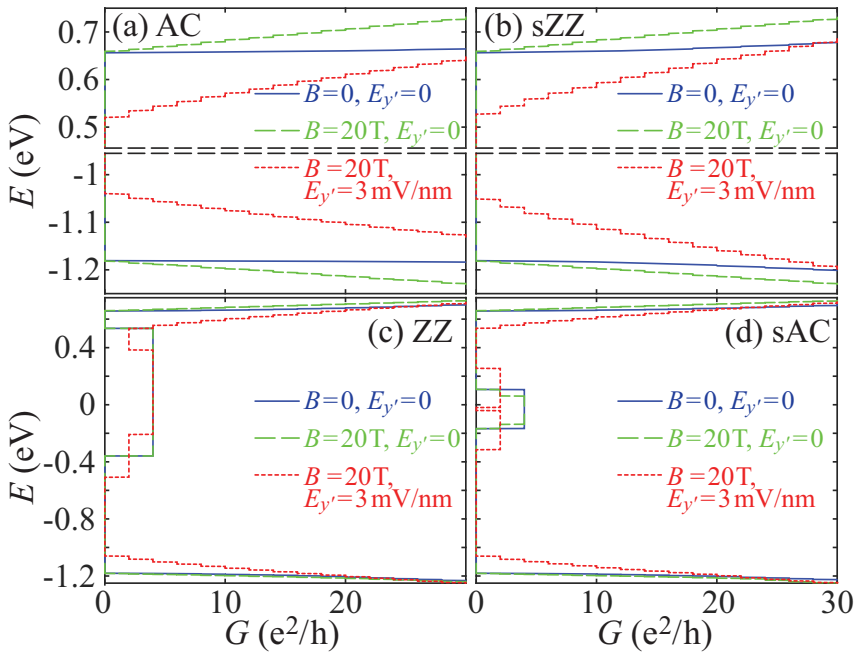

FIG. 10. The conductances of $N_{\mathrm{AC}}=610, N_{\mathrm{sZZ}}=380, N_{\mathrm{ZZ}}=$ 460 , and $N_{\mathrm{sAC}}=740$ are shown in the upper panel in (a), (b), (c), and (d), respectively. The dependences when fields are off are marked by solid blue lines, when only $B=20 \mathrm{~T}$ is shown by green dashed lines, and when $B=20 \mathrm{~T}$ and $E_{y^{\prime}}=3 \mathrm{mV} / \mathrm{nm}$ is displayed by dotted red lines.

field $E_{y^{\prime}}=1 \mathrm{mV} / \mathrm{nm}$ are applied. Since the energy spectra are dense in these wider ribbons, states originating from only the valence-band top and the conduction-band bottom, which are both located in the vicinity of the $\Gamma$ point, are displayed in this figure. As a priori inferred, wider ribbons are more suitable for our analytical estimates, which is confirmed by a comparison of the results of the analytical model and the obtained numerical results for this case. The analytical model predicts that a large number of Landau states emerge in AC ribbons, which are represented by linear segments in Figs. 9(a) and 9(e). As Figs. 9(b) and 9(f) show, the number of LLs in sZZ nanoribbons is comparably smaller. Yet it is larger than the number of LLs in ZZ nanoribbons, as Figs. 9(c) and 9(g) show. And similar to the latter case, a small influence of the magnetic field on sAC nanoribbons is found [see Figs. 9(d) and 9(h)]. The small difference between the sAC and $\mathrm{ZZ}$ nanoribbons is a consequence of the small angular separation between the sAC and $\mathrm{ZZ}$ directions. It also implies that the values of the parameters of the effective model are similar for the two directions.

For the considered nanoribbons, we compute the conductance for $T=0 \mathrm{~K}$ and display it in Fig. 10 for four types of edges. They all exhibit conductance quantization in a magnetic field. For the cases of AC and sZZ nanoribbons there are no edge states, so we show only conductance for a range of energies near the bottom (top) of the conduction (valence) band in Figs. 10(a) and 10(b). It is quite apparent that conductance adopts a distinctive staircase shape in a range of energies in which LLs exist. Furthermore, the diagram for a sufficiently large magnetic field (dashed green line) is considerably misplaced with respect to the $B=0$ case (solid blue line). The effects of a magnetic field on the conductance dependence are much smaller for the cases of $\mathrm{ZZ}$ and sAC nanoribbons, which are shown in Figs. 10(c) and 10(d). Here, only a few LLs are formed near the bottom (top) of the conduction (valence) for $B=20 \mathrm{~T}$. Applying a transverse electric field $E_{y^{\prime}}$ together with the magnetic field leads to a decrease of the band gap and an increase in the height of the stairs in the $G(E)$ dependence, as shown by red dotted lines in Fig. 10. It is a result consistent with Eq. (17). At higher energies, where LLs cease to exist, the separation between the levels is similar to the $B=0$ case.

One important detail in Fig. 10 should not escape our attention. It is the region of a constant conductance in the gap of the $\mathrm{ZZ}$ and sAC nanoribbons [see Figs. 10(c) and 10(d)]. The nonzero conductance here arises from the edge states hosted by these types of nanoribbons. This region exists for a zero magnetic field, and in the $\mathrm{ZZ}$ nanoribbons it is not affected by an applied magnetic field, as the overlap between the solid blue and dashed green lines in Fig. 10(c) indicates. Similarly, the central region is slightly modified in SAC nanoribbons [see Fig. 10(d)]. But, when both the electric and magnetic fields are applied, the conductance changes considerably. These changes are established by means of splitting and shifting of the edge states toward the bottom of the conduction and the top of the valence band. The eigenenergies of the edge states in $\mathrm{ZZ}$ nanoribbons vary considerably with momentum and decrease the band gap. Hence $\mathrm{ZZ}$ nanoribbons are metallic regardless of width, which is a detail that might be derived from Fig. 10(c). On the other hand, in sAC nanoribbons the band gap opens around the Fermi level even for a small value of $E_{y^{\prime}}$, which turns the nanoribbon into an insulating state. We might conjecture that this effect could be useful for efficient lateral gating of field-effect transistors (FET) with a channel made of a single-layer phosphorus sheet [15].

\section{SUMMARY AND CONCLUSIONS}

In summary, we derived a Hamiltonian to describe the electronic structure of phosphorene nanoribbons with arbitrary edges in transverse electric and perpendicular magnetic fields. We found that when a magnetic field is turned on, the states of positive and negative momenta split to the opposite sides of the nanoribbon. An analytical expression for the minimal magnetic field when the bands become flat is obtained. The boundaries of these flat segments in momentum space are also described by analytical functions. We show that both the minimal field and the extension of the flatbands depend on the type of nanoribbon edge. Furthermore, when the magnetic field increases, the Landau level spectrum emerges first in an armchair nanoribbon and last in the zigzag nanoribbon of equal width. Thus, the transverse confinement of electrons is found to be weakest in the zigzag nanoribbons and strongest in the armchair nanoribbons. Moreover, the application of an in-plane electric field causes the band gap to decrease and turns the previously flat segments into ranges of linear variation of the energy spectra in momentum space. We found that the electric field gives rise to shifts of crossings toward the center of the phosphorene Brillouin zone. Furthermore, the analytical expression for the critical magnetic field, when the edge states that are localized at opposite edges acquire counter phases, is derived for the cases of zigzag and skewed armchair nanoribbons. For all analyzed nanoribbon types and for two values of width, we found good agreement between the numerical results obtained by means of the tight-binding method and 
the results derived from an analytical model. Furthermore, due to quantization of Landau levels, conductance exhibits a characteristic staircase shape at low temperature, and stairlike features of conductance due to edge states are found in the band gap. The band gap in skewed armchair nanoribbons appeared to be efficiently opened and closed by an in-plane electric field, which might be important for nanoelectronic applications of phosphorene.

\section{ACKNOWLEDGMENT}

This work was supported by Erasmus+, the Serbian Ministry of Education, Science and Technological Development, and the Flemish Science Foundation (FWO-V1).

\section{APPENDIX: THE EFFECTIVE HAMILTONIAN}

Using the tight-binding model, the four-band Hamiltonian $[16,19]$ for a single-layer phosphorene sheet is given by

$$
H_{k}^{4 \times 4}=\left(\begin{array}{cccc}
U_{A} & H_{A B} & H_{A D} & H_{A C} \\
H_{A B}^{*} & U_{B} & H_{B D} & H_{B C} \\
H_{A D}^{*} & H_{B D}^{*} & U_{D} & H_{D C} \\
H_{A C}^{*} & H_{B C}^{*} & H_{D C}^{*} & U_{C}
\end{array}\right),
$$

with eigenvectors represented by the spinor $\left[\phi_{A} \phi_{B} \phi_{D} \phi_{C}\right]^{T}$, and diagonal terms are given by

$$
\begin{aligned}
U=U_{A-D}= & 2 t_{3} \cos \left(a_{x} k_{x}\right)+2 t_{7} \cos \left(a_{y} k_{y}\right) \\
& +4 t_{10} \cos \left(a_{x} k_{x}\right) \cos \left(a_{y} k_{y}\right) .
\end{aligned}
$$

The interaction terms between sublattice sites are

$$
\begin{gathered}
H_{A B}=H_{C D}^{*}=t_{2} e^{i k_{y} h_{2 y}}+t_{6} e^{-i k_{y}\left(a_{y}-h_{2 y}\right)} \\
+2 t_{9} \cos \left(a_{x} k_{x}\right) e^{-i k_{y}\left(a_{y}-h_{2 y}\right)} \\
H_{A D}=H_{B C}^{*}=4 t_{5} \cos \left(\frac{a_{x} k_{x}}{2}\right) \cos \left(\frac{a_{y} k_{y}}{2}\right) \\
H_{A C}=H_{B D}^{*}=2 t_{4} \cos \left(\frac{a_{x} k_{x}}{2}\right) e^{i k_{y}\left(a_{y}-h_{1 y}\right)} \\
+2\left[t_{1} \cos \left(\frac{a_{x} k_{x}}{2}\right)+t_{8} \cos \left(\frac{3 a_{x} k_{x}}{2}\right)\right] e^{-i k_{y} h_{1 y}}
\end{gathered}
$$

Here $h_{1 y}=d_{1} \cos \left(\alpha_{\text {hex }} / 2\right)$ and $h_{2 y}=d_{2} \cos \beta$ (see Fig. 1). The parameter values are taken from Ref. [5]. The equality of certain terms of the Hamiltonian (A1) indicates the "equivalence" of certain atomic sites. This is actually due to the $D_{2 h}$ point-group invariance [16,21], which is preserved even when a perpendicular magnetic field is applied. Atoms in the upper and lower lattice, namely $A \equiv D$ and $B \equiv C$, are "indistinguishable," and the unit cell is reduced to a single dimer, framed by the dashed square in Fig. 1. Using this symmetry argument, the simplified two-band Hamiltonian reads

$$
H_{\mathbf{k}}^{2 \times 2}=\left[\begin{array}{cc}
U+H_{A D} & H_{A B}+H_{A C} \\
H_{A B}^{*}+H_{A C}^{*} & U+H_{A D}
\end{array}\right],
$$

which acts upon the spinors

$$
\psi=\left[\begin{array}{c}
\left(\phi_{A}+\phi_{D}\right) / 2 \\
\left(\phi_{B}+\phi_{C}\right) / 2
\end{array}\right]=\left[\begin{array}{l}
\phi_{1} \\
\phi_{2}
\end{array}\right] .
$$

The eigenvalue problem for the Hamiltonian (A6) can be solved analytically,

$$
E=U+H_{A D} \pm\left|H_{A B}+H_{A C}\right|,
$$

where the upper (lower) sign is for the conduction (valence) band.

Recent theoretical analysis [16,19] based on the tightbinding model with five hopping parameters shows that the MacLaurin series of analytical functions in the vicinity of the $\Gamma$ point up to the quadratic term yield satisfactory accuracy for the phosphorene band structure. Using a similar argument, we derive the effective two-band Hamiltonian

$$
H_{\mathbf{k} \rightarrow \Gamma}^{2 \times 2}=\left[\begin{array}{cc}
f & g \\
g^{\dagger} & f
\end{array}\right],
$$

where $f=E_{0}+\chi_{x} k_{x}^{2}+\chi_{y} k_{y}^{2}$ and $g=E_{g} / 2-i \gamma k_{y}+\alpha_{x} k_{x}^{2}+$ $\alpha_{y} k_{y}^{2}$, with

$$
\begin{gathered}
E_{0}=2 t_{3}+4 t_{5}+2 t_{7}+4 t_{10}=-0.262 \mathrm{eV}, \\
E_{g}=4 t_{1}+2 t_{2}+4 t_{4}+2 t_{6}+4 t_{8}+4 t_{9}=1.838 \mathrm{eV}, \\
\chi_{x}=\left(-t_{3}-t_{5} / 2-2 t_{10}\right) a_{x}^{2}=0.01060 \mathrm{eV} \mathrm{nm}^{2}, \\
\chi_{y}=\left(-t_{5} / 2-t_{7}-2 t_{10}\right) a_{y}^{2}=-0.01772 \mathrm{eV} \mathrm{nm}^{2}, \\
\alpha_{x}=\left(-t_{1} / 4-t_{4} / 4-9 t_{8} / 4-t_{9}\right) a_{x}^{2}=0.0224 \mathrm{eV} \mathrm{nm}^{2},
\end{gathered}
$$

$$
\begin{aligned}
\alpha_{y}= & -\left(t_{1}+t_{8}\right) h_{1 y}^{2}-t_{2} / 2 h_{2 y}^{2}-t_{4}\left(a_{y}-h_{1 y}\right)^{2} \\
& -\left(t_{6} / 2+t_{9}\right)\left(a_{y}-h_{2 y}\right)^{2}=0.02026 \mathrm{eV} \mathrm{nm}^{2}, \\
\gamma= & 2\left(t_{1}+t_{8}\right) h_{1 y}-t_{2} h_{2 y}-2 t_{4}\left(a_{y}-h_{1 y}\right) \\
& +\left(t_{6}+2 t_{9}\right)\left(a_{y}-h_{2 y}\right)=-0.5952 \mathrm{eV} \mathrm{nm},
\end{aligned}
$$

and we obtain the simplified dispersion for electrons and holes,

$$
\begin{aligned}
E_{e(h)}= & E_{0}+\chi_{x} k_{x}^{2}+\chi_{y} k_{y}^{2} \\
& \pm \sqrt{\left(\frac{E_{g}}{2}+\alpha_{x} k_{x}^{2}+\alpha_{y} k_{y}^{2}\right)^{2}+\gamma^{2} k_{y}^{2} .}
\end{aligned}
$$

From Eq. (A17), it is obvious that phosphorene has a nonelliptical dispersion due to the parameter $\gamma$.

To transform the low-energy Hamiltonian given by Eq. (A9) into a more convenient form, we perform the unitary transformation given by the Hadamard matrix,

$$
U=\frac{1}{\sqrt{2}}\left[\begin{array}{cc}
1 & 1 \\
1 & -1
\end{array}\right] .
$$

The resulting Hamiltonian has the same form as the one obtained in Ref. [13] by fitting to results from $a b$ initio calculations,

$$
H_{\mathbf{k} \rightarrow \Gamma}^{U}=\left[\begin{array}{cc}
E_{c} & i \gamma k_{y} \\
-i \gamma k_{y} & E_{v}
\end{array}\right]
$$


where the diagonal terms

$$
\begin{aligned}
E_{c} & =E_{0}+\frac{E_{g}}{2}+\left(\alpha_{x}+\chi_{x}\right) k_{x}^{2}+\left(\alpha_{y}+\chi_{y}\right) k_{y}^{2} \\
& =E_{c 0}+\frac{\hbar^{2}}{2 m_{0 e, x}^{*}} k_{x}^{2}+\frac{\hbar^{2}}{2 m_{0 e, y}^{*}} k_{y}^{2}, \\
E_{v} & =E_{0}-\frac{E_{g}}{2}-\left(\alpha_{x}-\chi_{x}\right) k_{x}^{2}-\left(\alpha_{y}-\chi_{y}\right) k_{y}^{2} \\
& =E_{v 0}-\frac{\hbar^{2}}{2 m_{0 h, x}^{*}} k_{x}^{2}-\frac{\hbar^{2}}{2 m_{0 h, y}^{*}} k_{y}^{2},
\end{aligned}
$$

are related to the conduction- and valence-band dispersions, respectively, while the off-diagonal term introduces interband coupling. Here, $E_{c(v) 0}$ are the conduction (valence) -band edges in monolayer phosphorene, and $m_{0 e, x(y)}^{*}=\hbar^{2} / 2\left(\alpha_{x(y)}+\chi_{x(y)}\right)$ and $m_{0 h, x(y)}^{*}=\hbar^{2} / 2\left(\alpha_{x(y)}-\chi_{x(y)}\right)$ are the electron and hole effective masses when the coupling is neglected. Furthermore, spinors given by Eq. (A7) are transformed to $\psi^{U}=\left[\phi_{c} \phi_{v}\right]^{T}$, where $\phi_{c(v)}=\left(\phi_{1} \pm \phi_{2}\right) / \sqrt{2}$.

In the long-wavelength limit, $E_{c}-E_{v}>E_{g} \gg\left|\gamma k_{y}\right|$. Thus, the second term in Eq. (A17) is expanded around the $\Gamma$ point, and the energy dispersions of conduction and valence bands are approximated by $E_{c}^{\text {eff }}=E_{c}+\gamma^{2} k_{y}^{2} / E_{g}$ and $E_{v}^{\text {eff }}=E_{v}-\gamma^{2} k_{y}^{2} / E_{g}$, respectively [18]. Therefore, the Hamiltonian given by Eq. (A19) is reduced to the diagonal form $H_{k}^{\text {eff, } U} \rightarrow \operatorname{diag}\left(E_{c}^{\text {eff }}, E_{v}^{\text {eff }}\right)$.
[1] W. Lu, H. Nan, J. Hong, Y. Chen, C. Zhu, Z. Liang, X. Ma, Z. Ni, and C. Jin, Nano Res. 7, 853 (2014).

[2] H. Liu, A. T. Neal, Z. Zhu, Z. Luo, X. Xu, D. Tománek, and P. D. Ye, ACS Nano 8, 4033 (2014).

[3] L. Li, Y. Yu, G. J. Ye, Q. Ge, X. Ou, H. Wu, D. Feng, X. H. Chen, and Y. Zhang, Nat. Nanotech. 9, 372 (2014).

[4] S. P. Koenig, R. A. Doganov, H. Schmidt, A. H. C. Neto, and B. Özyilmaz, Appl. Phys. Lett. 104, 103106 (2014).

[5] A. N. Rudenko, S. Yuan, and M. I. Katsnelson, Phys. Rev. B 92, 085419 (2015); 93, 199906(E) (2016).

[6] R. Fei, A. Faghaninia, R. Soklaski, J.-A. Yan, C. Lo, and L. Yang, Nano Lett. 14, 6393 (2014).

[7] Y. Aierken, D. Çakir, C. Sevik, and F. M. Peeters, Phys. Rev. B 92, 081408 (2015).

[8] J. Qiao, X. Kong, Z.-X. Hu, F. Yang, and W. Ji, Nat. Commun. 5, 4475 (2014).

[9] A. Favron, E. Gaufrès, F. Fossard, A.-L. P.-L'Heureux, N. Y.-W. Tang, P. L. Lévesque, A. Loiseau, R. Leonelli, S. Francoeur, and R. Martel, Nat. Mater. 14, 826 (2015).

[10] Y. Cao, A. Mishchenko, G. L. Yu, E. Khestanova, A. P. Rooney, E. Prestat, A. V. Kretinin, P. Blake, M. B. Shalom, C. Woods, J. Chapman, G. Balakrishnan, I. V. Grigorieva, K. S. Novoselov, B. A. Piot, M. Potemski, K. Watanabe, T. Taniguchi, S. J. Haigh, A. K. Geim, and R. V. Gorbachev, Nano Lett. 15, 4914 (2015).

[11] L. Li, G. J. Ye, V. Tran, R. Fei, G. Chen, H. Wang, J. Wang, K. Watanabe, T. Taniguchi, L. Yang, X. H. Chen, and Y. Zhang, Nat. Nanotech. 10, 608 (2015).

[12] X. Chen, Y. Wu, Z. Wu, Y. Han, S. Xu, L. Wang, W. Ye, T. Han, Y. He, Y. Cai, and N. Wang, Nat. Commun. 6, 7702 (2015).

[13] A. S. Rodin, A. Carvalho, and A. H. C. Neto, Phys. Rev. Lett. 112, 176801 (2014).

[14] D. Çakir, H. Sahin, and F. M. Peeters, Phys. Rev. B 90, 205421 (2014).
[15] M. M. Grujić, M. Ezawa, M. Ž. Tadić, and F. M. Peeters, Phys. Rev. B 93, 245413 (2016).

[16] M. Ezawa, New J. Phys. 16, 115004 (2014).

[17] E. T. Sisakht, M. H. Zare, and F. Fazileh, Phys. Rev. B 91, 085409 (2015).

[18] X. Y. Zhou, R. Zhang, J. P. Sun, Y. L. Zou, D. Zhang, W. K. Lou, F. Cheng, G. H. Zhou, F. Zhai, and K. Chang, Sci. Rep. 5, 12295 (2015).

[19] J. M. Pereira, Jr. and M. I. Katsnelson, Phys. Rev. B 92, 075437 (2015).

[20] B. Ostahie and A. Aldea, Phys. Rev. B 93, 075408 (2016).

[21] L. C. L. Y. Voon, A. Lopez-Bezanilla, J. Wang, Y. Zhang, and M. Willatzen, New J. Phys. 17, 025004 (2015).

[22] A. N. Rudenko and M. I. Katsnelson, Phys. Rev. B 89, 201408(R) (2014).

[23] R. Winkler, in Spin-orbit Coupling Effects in Two-Dimensional Electron and Hole Systems (Springer, Berlin, 2003), pp. 164-166.

[24] D. B. Topalović, V. V. Arsoski, S. Pavlović, N. A. Čukarić, M.Ž. Tadić, and F. M. Peeters, Commun. Theor. Phys. 65, 105 (2016).

[25] D. J. P. de Sousa, L. V. de Castro, D. R. da Costa, and J. M. Pereira, Jr., Phys. Rev. B 94, 235415 (2016).

[26] S. Datta, in Electronic Transport in Mesoscopic Systems (Cambridge University Press, New Delhi, 2011), Chap. 3, Sec. 5, pp. 117-170.

[27] D. S. Fisher and P. A. Lee, Phys. Rev. B 23, 6851 (1981).

[28] M. M. Grujić, M. Ž. Tadić, and F. M. Peeters, Phys. Rev. B 91, 245432 (2015).

[29] M. Wimmer, PhD. thesis, Universität Regensburg (2008).

[30] M. Grujić, PhD. thesis (joint), University of Belgrade, School of Electrical Engineering and University of Antwerp, Department of Physics (2015). 